\title{
Relación entre el cumplimiento del procedimiento de aspiración de secreciones y la presencia de complicaciones en pacientes críticos, emergencia de un hospital nacional de Lima
}

\author{
Diana Uceda-Ochoa ${ }^{1, \mathrm{a}}$, Patricia Obando-Castro ${ }^{2, \mathrm{~b}}$
}

\begin{abstract}
RESUMEN
Objetivo: determinar cuál es la relación entre el cumplimiento del procedimiento de aspiración de secreciones y la presencia de complicaciones en pacientes adultos en la sala de pacientes críticos del servicio de emergencia del Hospital Nacional Guillermo Almenara Irigoyen. Material y método: el estudio fue observacional de enfoque cuantitativo de alcance descriptivo. Los datos fueron recolectados en dos listas de cotejo, la primera recogió información sobre el cumplimiento de los pasos que realizó el profesional de enfermería en el procedimiento de aspiración de secreciones y la segunda recogió información sobre las complicaciones que se presentaron en el paciente durante el procedimiento: hipoxia, arritmia cardiaca, hipotensión y lesión de la mucosa traqueal. Análisis de datos: se realizó mediante estadística descriptiva (frecuencia y porcentaje) y estadística inferencial para establecer la relación entre variables (chi cuadrado). Resultados: el nivel de cumplimiento fue de medio a bajo en un 60\%. Las complicaciones más frecuentes fueron lesión de la mucosa traqueal con un $65 \%$, hipoxia con un $55 \%$. Se encontró relación estadísticamente significativa entre el nivel de cumplimiento bajo y la presencia de lesión de la mucosa traqueal y la hipoxia $(\mathrm{P}<0.05)$. Conclusión:
\end{abstract}

Palabras clave: cuidado de enfermería, complicaciones.

\begin{abstract}
The goal was to determine what is the relationship between compliance with the procedure of aspiration of secretions and the presence of complications in adult patients in the room of critical patients from emergency service of the Guillermo Almenara Irigoyen National Hospital. Material and method: the study was observational approach to quantitative descriptive scope. The data were collected in two lists of matching, the first collected information on the implementation of the steps made by the nursing professional in the procedure of aspiration of secretions and the second collected information about the complications that arose in the patient during the procedure: hypoxia, cardiac arrhythmia, hypotension, and tracheal mucosal injury. Data analysis: was performed by descriptive statistics (frequency and percentage) and statistical inference to establish the relationship between variables (Chi-square). Results: The level of compliance was of medium to low at $60 \%$. The most frequent complications were lesion of the tracheal mucosa with $65 \%$, hypoxia with $55 \%$. It was found statistically significant relationship between low compliance level and the presence of lesion of the tracheal mucosa and hypoxia $(\mathrm{P}<0.05)$.
\end{abstract}

Key words: nursing care, complications.

Hospital Nacional Guillermo Almenara Irigoyen. Lima, Perú.

Centro Medico Naval. Lima, Perú. 


\section{INTRODUCCION}

La Sala de Pacientes Críticos del Servicio de Emergencia del Hospital Nacional Guillermo Almenara Irigoyen, tiene capacidad para 15 pacientes adultos de los cuales un 20 a $30 \%$ de ellos son portadores de una vía aérea artificial que puede ser: tubo orotraqueal o tubo de traqueostomia, por lo que dentro del plan de cuidados la realización del procedimiento de aspiración de secreciones es frecuente en dicha unidad y es responsabilidad del profesional de enfermería mantener la vía aérea permeable mediante el aseguramiento de la calidad del cuidado por lo que es necesario monitorizar la ejecución de los procesos involucrados en la atención del paciente para el logro de los objetivos.

El procedimiento de aspiración de secreciones es una técnica que consiste en extraer las secreciones bronquiales de las vías respiratorias bajas para mantenerlas permeables. Dado que la respiración involucra procesos vitales como el intercambio gaseoso en los pulmones, que resulta indispensable para el metabolismo celular, la instalación y el mantenimiento de una vía aérea artificial, como el tubo orotraqueal o cánula de traqueostomia (1).

La presente investigación, esta refrendada por la teoría de la Atención Burocrática de Marilin Anne Ray quien define a la profesión de enfermería como un cuidado holístico, espiritual y ético que busca el bien propio y ajeno en complejas comunidades, organizaciones y culturas burocráticas, teniendo implicancia directa para le enfermera clínica (asistencial) y la enfermera gestora, las primeras, se ven obligadas a integrar conocimientos y habilidades en los cuidados que realiza (2).

La Gestión del Cuidado de Enfermería, se define como la aplicación de un juicio, organización, motivación y control de la provisión de cuidados, oportunos, seguros, integrales, que aseguren la continuidad de la atención y se sustenten en lineamientos estratégicos (3).

El cuidado no solo se limita a satisfacer las necesidades físicas más inmediatas, según Colliere el cuidar es el acto que representa una infinita variedad de actividades dirigidas a mantener y conservar la vida y a facilitar las condiciones para la realización del proceso vital humano, el cuidado implica comprender las necesidades de la otra persona y ello significa un proceso de empatía, identificación y proyección; también como fenómeno humano el cuidado tiene la dimensión de la subjetividad, la cual precisa de apertura de la voluntad y el afecto de la persona (4).
Así mismo cuidar requiere conocer a cada persona, interesarse por él, interactuar de forma que nos permita desarrollar el vínculo afectivo transpersonal, es decir ponerse en el lugar de la otra persona, saber lo que siente y piensa acerca de su bienestar o enfermedad, es importante mencionar en el trabajo a investigar, que el procedimiento de aspiración de secreciones es sólo una parte del cuidado que brinda el enfermería para prevenir alguna complicación para el beneficio y el bienestar de la persona (5).

El cuidado de enfermería debe ser por lo tanto integral, es decir no sólo lo físico sino también lo biopsicosocial y espiritual, además debe ser eficiente y producir impacto en la salud del individuo y comunidad, por lo que el profesional de enfermería está preparado de manera íntegra conoce el contexto social, político, económico del país, la situación de salud del usuario, los sistemas de apoyo, creencias, valores, estilos de vida y metas relacionados con la salud, de acuerdo con la legislación vigente (6).

\section{Material y métodos:}

El estudio fue de enfoque cuantitativo de alcance descriptivo y observacional. La población estuvo constituida por 20 enfermeras (os) que trabajan en la Sala de Pacientes Críticos del servicio de emergencia del Hospital Nacional Guillermo Almenar Irigoyen, durante los meses de Enero a Marzo del 2013.

Para la recolección de datos se utilizó una lista de cotejo que contiene los pasos del procedimiento de aspiración de secreciones: antes, durante y después del procedimiento.

Se identificó a los 20 enfermeros, cada enfermero se le asignó un paciente, siendo la población de estudio el profesional de enfermería que labora en la sala de pacientes críticos del servicio de emergencia del Hospital Nacional Guillermo Almenara Irigoyen.

Se consideró que la enfermera (o) si cumplió con el procedimiento cuando ejecuto, o cumplió con todos los pasos contenidos en el instrumento, la cual consta de 16ítems; cada ítem tiene dos alternativas "SI" si cumple con la acción del enunciado y "NO" si no cumple con la acción del enunciado. También se midió el nivel de conocimiento bajo, medio o alto.

Este instrumento fue elaborado por la investigadora tomando como referencia el instrumento de auditoría: Aspiración de secreciones traqueo bronquiales aplicado en el Instituto Nacional de Cardiología Ignacio Chávez. Subdirección de enfermería de México, la cual fue validada a través del juicio de expertos. 
Como segundo instrumento será una lista de cotejo donde se registró datos del paciente y consta de dos partes: la primera son los datos generales del paciente y la segunda parte los datos clínicos como el tipo de vía aérea, saturación de oxígeno, así como el control de funciones vitales al inicio, durante y al final del procedimiento. Finalmente se registró la presencia de complicaciones durante la ejecución del procedimiento de aspiración de secreciones.

La validación del instrumento, se realizó a través del juicio de expertos mediante el cual se consultó a 10 expertos en el tema.

Se solicitó la autorización del Comité de Ética de la Universidad y del Comité de Ética e Investigación del Hospital Nacional Guillermo Almenara Irigoyen donde se realizó el trabajo de investigación.

Luego de recolectada la información, previo control de calidad, se procedió al almacén de la información en una base de datos creada en el Programa Estadístico SPSS (Statistical Program For Social Science) versión 19.0 para Windows para su análisis.

\section{RESULTADOS}

Tabla 1. Nivel del cumplimiento del procedimiento de aspiración de secreciones en la sala de pacientes críticos del Hospital Nacional Guillermo Almenara Irigoyen enero a marzo del 2013

\begin{tabular}{ccc}
\hline $\begin{array}{c}\text { Nivel de } \\
\text { cumplimiento }\end{array}$ & $\begin{array}{c}\text { NUMERO } \\
\mathrm{n}^{\circ}\end{array}$ & $\%$ \\
\hline BAJO & 5 & 25,0 \\
MEDIO & 7 & 35,0 \\
ALTO & 8 & 40,0 \\
Total & 20 & 100,0 \\
\hline
\end{tabular}

La tabla muestra que del $100 \%$ de los profesionales de enfermería el $40 \%$ tienen un nivel de cumplimiento alto, y el $25 \%$ presentan un nivel bajo.

Tabla 2 Nivel de cumplimiento antes del procedimiento de aspiración de secrecionesen la sala de pacientes críticos del Hospital Guillermo Almenara Irigoyen enero a marzo del 2013

\begin{tabular}{l|cccc}
\hline $\begin{array}{l}\text { CUMPLIMIENTO DEL PROCE- } \\
\begin{array}{l}\text { DIMIENTO DE ASPIRACIÓN } \\
\text { DE SECRECIONES }\end{array}\end{array}$ & \multicolumn{2}{c}{$\begin{array}{c}\text { SI } \\
\text { CUMPLE }\end{array}$} & \multicolumn{2}{c}{$\begin{array}{c}\text { NO } \\
\text { CUMPLE }\end{array}$} \\
\cline { 2 - 6 } & $\mathbf{n}$ & $\mathbf{\%}$ & $\mathbf{n}$ & $\mathbf{\%}$ \\
\hline $\begin{array}{l}\text { Verifica las conexiones del sistema } \\
\text { de aspiración. }\end{array}$ & $\mathbf{2 0}$ & $\mathbf{1 0 0 . 0}$ & 0 & 0 \\
$\begin{array}{l}\text { Verifica la programación de la } \\
\text { presión del aspirador. }\end{array}$ & 17 & 85.0 & 3 & 15.0 \\
$\begin{array}{l}\text { Posición del paciente en } 45^{\circ} \\
\text { Hiperoxigena al paciente }\end{array}$ & 19 & 95.0 & 1 & 5.0 \\
\hline
\end{tabular}

Se observar que el $100 \%$ de los profesionales de enfermería cumplen con la verificación de las conexiones del sistema de aspiración de secreciones, en tanto que el 55\% de enfermeros, no cumplen con hiperoxigenar al paciente.

Tabla 3 Nivel del cumplimiento del procedimiento de aspiración de secreciones durante la intervención de enfermería en la sala de pacientes críticos del

Hospital Nacional Guillermo Almenara Irigoyen enero a marzo del 2013

\begin{tabular}{l|cccc}
\hline $\begin{array}{l}\text { CUMPLIMIENTO DEL PROCE- } \\
\text { DIMIENTO }\end{array}$ & \multicolumn{2}{|c}{ SI } & \multicolumn{2}{c}{ NO } \\
CEMPLE & CUMPLE \\
DEIRACIÓN DE SECRE- & & & & \\
\cline { 2 - 6 } CIONES & $\mathbf{n}$ & $\mathbf{\%}$ & $\mathbf{n}$ & $\mathbf{\%}$ \\
\hline Guantes & 20 & 100.0 & 0 & 0 \\
Mascarillas & 19 & 95.0 & 1 & 5.0 \\
Lentes & 8 & 40.0 & 12 & 60.0 \\
Gorro & 15 & 75.0 & 5 & 25.0 \\
Mandilón & 19 & 95.0 & 1 & 5.0 \\
Lavado de manos & 20 & 100.0 & 0 & 0 \\
$\begin{array}{l}\text { Secuencia de la aspiración boca- } \\
\text { tubo }\end{array}$ & 5 & 25.0 & 15 & 75.0 \\
Tiempo de aspiración por pasada & 9 & 45.0 & 11 & 55.0 \\
menos de 10 segundos. & & & & \\
Utiliza diferentes sondas para boca & 15 & 75.0 & 5 & 25.0 \\
y tubo & & & & \\
Monitoriza permanentemente al & 17 & 85.0 & 3 & 15.0 \\
paciente & & & & \\
Verifica la presión arterial & 16 & 80.0 & 4 & 20.0 \\
Control de frecuencia cardiaca & 15 & 75.0 & 5 & 25.0 \\
Control de frecuencia respiratoria & 14 & 70.0 & 6 & 30.0 \\
\hline
\end{tabular}

Se observa que el $100 \%$ de los profesionales de enfermería cumplen con el uso de guantes y con el lavado de manos, de otro lado el $75 \%$ de los profesionales de enfermería no cumplen con la secuencia de la aspiración boca-tubo. 
Tabla 4 Nivel del cumplimiento del procedimiento de aspiración de secreciones durante la intervención de enfermería en la sala de pacientes críticos del

Hospital Nacional Guillermo Almenara Irigoyen enero a marzo del 2013

CUMPLIMIENTO DEL PROCE-
DIMIENTO
DE ASPIRACIÓN DE SECRE-
CIONES

Valora al paciente

Ausculta campos pulmonares

Evalúa el estado neurológico

Verifica cianosis distal

Registra en la historia clínica las

características de las secrecio-

nes:

- Color

- Volumen

- Cantidad

Eventos sucedidos durante el procedimiento

Se observa que del 100\% de profesionales de enfermería, el $75 \%$ cumplen con valorar a su paciente asignado, por otro lado se puede observar que un 50\% del grupo de enfermeros que no cumplen con registrar en la historia clínica las características de las secreciones después del procedimiento de aspiración de secreciones.

Tabla 5 Complicaciones en la ejecución del procedimiento de aspiración de secreciones en la sala de pacientes críticos del Hospital Guillermo Almenara Irigoyen enero a marzo del 2013

\begin{tabular}{lcc}
\hline $\begin{array}{l}\text { COMPLICACIONES } \\
(\mathrm{n}=20)\end{array}$ & NÚMERO & $\%$ \\
Lesión mucosa traqueal & 13 & 5.0 \\
Hipoxia & 11 & 55.0 \\
Arritmia & 5 & 20.0 \\
Hipotensión & 2 & 10.0 \\
\hline
\end{tabular}

Se observa que del $100 \%$, el $65 \%$ de los pacientes presentaron lesión de la mucosa traqueal, de otro lado un 55\% de los pacientes presentaron hipoxia.

Tabla 6 Relación entre el nivel del cumplimiento del procedimiento de aspiración de secreciones y la presencia de complicaciones en la sala de pacientes críticos del Hospital Nacional Guillermo Almenara Irigoyen enero a marzo 2013

\begin{tabular}{|c|c|c|c|c|c|c|c|}
\hline \multirow[t]{3}{*}{ conenchón } & \multicolumn{6}{|c|}{ 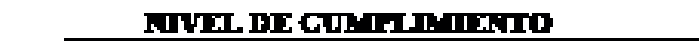 } & \multirow[t]{3}{*}{$\mathbf{F}$} \\
\hline & \multicolumn{2}{|r|}{ HASO } & \multicolumn{2}{|c|}{ MIDNO } & \multicolumn{2}{|c|}{ ALTO } & \\
\hline & $\mathbf{s}$ & *i & $\mathbf{F}$ & *it & $\mathbf{F}$ & wi & \\
\hline \multicolumn{8}{|l|}{ HIFAXXA } \\
\hline $\mathbf{N O}$ & $\mathbf{2}$ & $40,0 \%$ & $\mathbf{3}$ & $426 x$ & 4 & $50.0 \%$ & 1.00 \\
\hline Total & 5 & 10000 & 7 & $100,0 \%$ & ! & $100,0 \times$ & \\
\hline \multicolumn{8}{|l|}{ ARRTTMII } \\
\hline SI & $\mathbf{z}$ & (40) & $\mathbf{2}$ & $2 ; 0 x$ & $\mathbf{1}$ & $12,5 \%$ & 055 \\
\hline No & 3 & 40. & 5 & $71,4 \times 6$ & 7 & $\mathbf{7 3}, 5 \%$ & \\
\hline Tntal & $\mathbf{s}$ & $100,0 \times$ & $\mathbf{7}$ & $100,0 x$ & $\mathbf{z}$ & 10000 & \\
\hline \multicolumn{8}{|l|}{ BIPGIHM⿻上丨́ } \\
\hline SI & $\mathbf{1}$ & 2000 & $\mathbf{1}$ & $143 x$ & $\mathbf{a}$ &, 006 & 0.495 \\
\hline $\mathbf{m o}$ & 4 & $\cos$ & 5 & $5,7 \times$ & $n$ & $100.0 \%$ & \\
\hline Total & 5 & $100,0 \%$ & 7 & 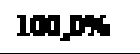 & $\mathbf{8}$ & $100,0 x$ & \\
\hline \multicolumn{8}{|l|}{ LESTín MDCOSA } \\
\hline SI & $\mathbf{s}$ & $100,0 \%$ & 5 & $25,7 x$ & $\mathbf{2}$ & 25,06 & \\
\hline No & D & איא & $\mathbf{1}$ & $14,3 \%$ & 5 & $75,5 \%$ & Doos \\
\hline Tatol & 5 & $100,0 x$ & 7 & 1000 & I & $100,0 x$ & \\
\hline
\end{tabular}


Se observa que del total de enfermeros, el $60 \%$ presentaron nivel de cumplimiento bajo y la complicación que presentó fue la hipoxia, mientras que para el grupo que presentó nivel de cumplimiento alto 4 de los profesionales, un $50 \%$ presentó la complicación. La diferencia no resulto estadísticamente significativa $(\mathrm{p}=1.00)$.

También se observa que para el grupo de enfermeros que presento nivel de cumplimiento bajo en el procedimiento de aspiración de secreciones, del total de enfermeros 2 de ellos, que equivale a un $40 \%$ se le complicó por arritmias, su paciente asignado, mientras que para el grupo que presentó nivel de cumplimiento alto, dicho porcentaje fue solo del $12.5 \%$, sin embargo la diferencia no resulto estadísticamente significativa $(\mathrm{p}=0.558)$.

En la misma tabla se observa que para el grupo de enfermeras que presentaron nivel de cumplimiento bajo en el procedimiento de aspiración de secreciones del total de enfermeros (100\%) un 20\% se complicó por hipotensión, su paciente asignado, mientras que para el grupo que presento nivel de cumplimiento alto, no se presentó ninguna complicación $(0 \%)$, sin embargo la diferencia no resulto estadísticamente significativa $(\mathrm{p}=0.495)$.

\section{DISCUSIÓN}

Entre los resultados encontrados se puede apreciar que en la tabla 1, el nivel de cumplimiento del procedimiento de aspiración de secreciones tuvo un $60 \%$ entre el nivel medio y bajo de dicho procedimiento; estos resultados podrían estar relacionados con la investigación realizada por Villanueva Cárdenas, en el año 2006, en donde la mayoría de los profesionales de enfermería en estudio tienen un nivel de conocimiento bajo; la práctica que desarrollan es adecuada antes del procedimiento; inadecuada durante el procedimiento y adecuada después del procedimiento de aspiración de secreciones en pacientes intubados (7).

De otro lado en el trabajo realizado por De Sousa, en su estudio sobre técnicas de aspiración de secreciones bronquiales que realiza el profesional de enfermería en la unidad de cuidados intensivos, también concluye que la población estudiada tiene conocimientos sobre dicha técnica; sin embargo se evidenció que durante la ejecución del procedimiento no se realiza en su totalidad los pasos a seguir (8). Probablemente esto se deba a que el profesional de enfermería obvia algunos pasos en el antes, durante o después del procedimiento sin tomar en cuenta la importancia de no ejecutar uno de los pasos pudiendo traer como consecuencia alguna complicación: hipoxia, arritmia cardiaca, hipotensión y la lesión de la mucosa traqueal, que puede afectar la salud del paciente al no llevar con efectividad dicho procedimiento, a pesar de los años de experiencia que tiene el profesional de enfermería a veces obvia algunos pasos tal vez por la falta de tiempo, la sobre demanda de pacientes y la recarga laboral hace que el profesional de enfermería encargado del procedimiento tenga que dejar de ejecutar alguno de los pasos, según lo estipulado para este procedimiento; tal y como lo demuestra la tabla 2 , existe un $100 \%$ de enfermeros que si cumplen con verificar las conexiones del sistema de aspiración antes del procedimiento pero existe un $55 \%$ enfermeros que no hiperoxigenan al paciente antes de la aspiración de secreciones lo que puede traer como complicación hipoxia con de saturación de oxígeno.

En pacientes intubados y con ventilación mecánica, la hiperoxigenación debe hacerse antes y después de la aspiración de secreciones. La hiperoxigenación en pacientes conectados al ventilador mecánico puede ser mantenida por dos minutos, ya que el ventilador entrega más altos niveles de oxígeno de las que se obtienen con la ventilación manual (9).

Sin embargo en aquellos pacientes que respiran espontáneamente, la hiperoxigenación es obtenida aumentando el aporte de oxígeno y educando al paciente a que respire profundamente. El éxito depende de la capacidad del paciente para aumentar la capacidad pulmonar. Si el paciente no puede respirar profundamente debe ser asistido con una bolsa de ventilación manual (10).

Así mismo observamos en la tabla 3, con respecto al cumplimiento durante el procedimiento de aspiración de secreciones que del $100 \%$ de la población, utilizan guantes para la ejecución del procedimiento como barrera de bioseguridad, pero de otro lado existe un porcentaje $75 \%$ enfermeros que no cumplen con la secuencia de la aspiración boca-tubo y tampoco se toma en cuenta el tiempo de duración de la aspiración de secreciones.

La succión de la vía aérea no debe extenderse más de 10 segundos pasado este tiempo el paciente está en riesgo de presentar hipoxemia por lo que es necesario hiperoxigenarlo antes de realizar la succión aumentando de esta manera la concentración de oxígeno al $100 \%$ de 3 a 5 minutos, por lo que es necesario hiperoxigenar al paciente antes de realizar la aspiración de secreciones (11).

La tabla 4, sin embargo nos demuestra que existe un $75 \%$ enfermeros, que si valoran el estado en que se encuentra el apaciente después del procedimiento de aspiración de secreciones; pero también existe un $50 \%$ de enfermeros que no registran en la historia clínica las características de las secreciones, se puede atribuir también a la falta de tiempo, 
sobrecarga laboral, que se obvia en el momento que se ejecuta el procedimiento, tal y como no los describe Weiss Orzuelos en su trabajo investigado Cuidados intensivos durante el procedimiento de aspiración endotraqueal y traqueostomia realizado en una unidad de cuidados intensivos en un hospital del Brasil; en donde existe alrededor de un $94.7 \%$ que no realizó la auscultación pulmonar y que tampoco lo registró en la Historia clínica (12).

Sin embargo el grupo en estudio se olvidan o no tienen tiempo para registrar en la historia clínica, las características de la aspiración de secreciones en las notas de enfermería.

En la tabla 5, se puede observar que la complicación más frecuente es la lesión de la mucosa traqueal, seguida de la hipoxia. El trauma de la mucosa puede deberse a las técnicas inapropiadas como puede ser la inserción de la sonda de aspiración demasiado profunda o la aplicación de presión negativa elevada durante la introducción de la misma que puede favorecer el sangrado de la mucosa traqueal. Lo indicado para la aspiración de secreciones es una presión que va entre 80 hasta $120 \mathrm{mmHg}$. Tal como lo manifiesta De Sousa que durante el procedimiento de aspiración de secreciones, el profesional de enfermería no realiza en su totalidad los pasos de esta técnica y que a su vez no existe un criterio único para realizar los pasos correctos de la misma, lo cual podría ocasionar graves complicaciones en el paciente (8).

Al hacer una comparación entre los estudios realizados tal como se observa en la Tabla 6 sobre la relación entre el nivel del cumplimiento del procedimiento de aspiración de secreciones y la presencia de complicaciones, se puede confirmar que aún existe un alto porcentaje de profesionales de enfermería que tienen un nivel bajo en el nivel del cumplimiento de este procedimiento, probablemente haga falta capacitación o mejorar las guías de atención para favorecer la promoción de los cuidados de enfermería oportunos y eficientes para de esta manera disminuir la posibilidad de cometer errores asociados al trabajo diario del profesional de enfermería, y evitar las complicaciones, como se observa en la tabla la de mayor porcentaje es la lesión de la mucosa traqueal.

Así mismo, en el estudio realizado por Collado Cabañin, plantea propuestas de capacitación para mejorar la gestión del cuidado en la aspiración de secreciones ya que del nivel de competencia de los enfermeros dependerá la calidad de la gestión del cuidado de enfermería (13).

\section{REFERENCIAS BIBLIOGRAFICAS}

1. González M, Restrepo G. Corporación para investigaciones biológicas. Medellín Colombia; 2006. p. 368.

2. Marriner a. Modelos y teorías en enfermería. $6^{\circ}$ ed. españa; 2007.

3. Waldow V. Cuidado humano. Brasil: editorial sagra luzzatto; 1998; 56-62.

4. Boff L. Saber cuidar. Ética do humano-compaisano pela tena. Brasil: editora lozes; 1999. p. 90-92.

5. Colliere M. Promover la vida. madrid: interamericana; 1993. p. 235.

6. Chalela S. Actualización en enfermería. vol. $8 \mathrm{n}^{\circ} 1$. Colombia; 2006. p. 6.

7. Villanueva. Nivel de conocimientos y prácticas de las enfermeras sobre la aspiración de secreciones en pacientes intubados en el servicio de emergencia y la unidad de cuidados intensivos del hospital huaura oyón. lima; 2006.

8. De sousa, m. Técnicas de aspiración de secreciones bronquiales que realiza el personal de enfermería. clínica razetti de barquisimeto: unidad de cuidados intensivos, lara - Venezuela; 2012.

9. Sociedad Argentina de terapia intensiva. ventilación mecánica .argentina: médica panamericana 2008.

10.Clemente F. Ventilación mecánica-guía para enfermería. sociedad española de cuidados respiratorios y tecnologías aplicadas. $1^{\mathrm{a}}$ ed. españa: elsevier; 2009.

11. Ministerio de salud. Norma general administrativa ${ }^{\circ}$ 19. gestión del cuidado de enfermería para la atención cerrada. ${ }^{\circ}$ 1127. Santiago: Minsa; 2007

12. Weiss S. Cuidados intensivo durante el procedimiento de aspiración endotraqueal y traqueotomía en la unidad de cuidados intensivos del hospital general de maternidad ramos tereza. Brasil; 2010.

13. collado Cabañin L. Propuestas de capacitación para mejorar la gestión del cuidado en la aspiración endotraqueal. cuba: hospital pediátrico docente "José Luis miranda". universidad de ciencias médicas de villa clara; 2009.

Fecha de recibido: 10 de Octubre de2014

Fecha de aceptación: 25 de Noviembre de 2014. 